\title{
The molecular structure of a dodecanol-graphite interface defects shov by STM: Imaging of pure 1-dodecanol and 1-dodecanol-1,8-octanediol adsorbed layers
}

\author{
Jean-Claude Poulin
}

Laboratoire de Synthèse Asymétrique (UA CNRS 1497), Institut de Chimie Moléculaire d'Orsay, Université de Paris Sud, 91405 Orsay Cedex, France

(Received July 4; accepted October 26, 1994)

\begin{abstract}
Résumé . - Létude par STM d'une couche de dodécanol adsorbée sur graphite montre la présence d'une proportion importante d'anomalies de jonctions entre alignements moléculaires. Une observation attentive révèle que ces défauts sont tous équivalents, sans réussir à déterminer le type des jonctions erronées par variation des paramètres STM. La visualisation d'une couche de dodécanol coadsorbé avec de l'octanediol a permis la distinction entre les deux extrémités du dodécanol, donc la détermination de la structure au niveau des défauts d'organisation de la couche.
\end{abstract}

\begin{abstract}
The STM observation of a dodecanol layer adsorbed on graphite has revealed a large amount of boundary defects between lamellae. A careful analysis of STM views showed the equivalency of defects, but a screening of tunnelling parameters was not able to determine whether irregular junctions are between methyl or hydroxyl termini of molecules. The observation of a mixed layer of coadsorbed dodecanol and octanediol showed the difference between molecular bounds, so the structure of defects was revealed.
\end{abstract}

\section{Introduction.}

The scanning tunnelling microscope (STM) is a powerful tool for observations at the atomic or molecular scale. The initial field of STM visualisations was semiconductors or metal surfaces in ultrahigh vacuum. Organic molecules are mainly electric insulators. Their STM study must be performed on a specially prepared sample by deposition onto a flat and conductor substrate, usually near room temperature and in air atmosphere. For small to medium sized molecules, the adsorbed layers are generally found to be organized at the observed interface. A long linear alkyl chain is considered as a favorable part of a molecule for STM visualisation, at least on graphite. Some of the observed adsorbates (see reviews in Refs. [1] and [2]) in air atmosphere are: long chain linear alkanes, fatty acids, primary alcohols, polyalkyl benzene and liquid crystals.

Scanning tunnelling microscopy is well suited for the direct characterisation of the structure of 

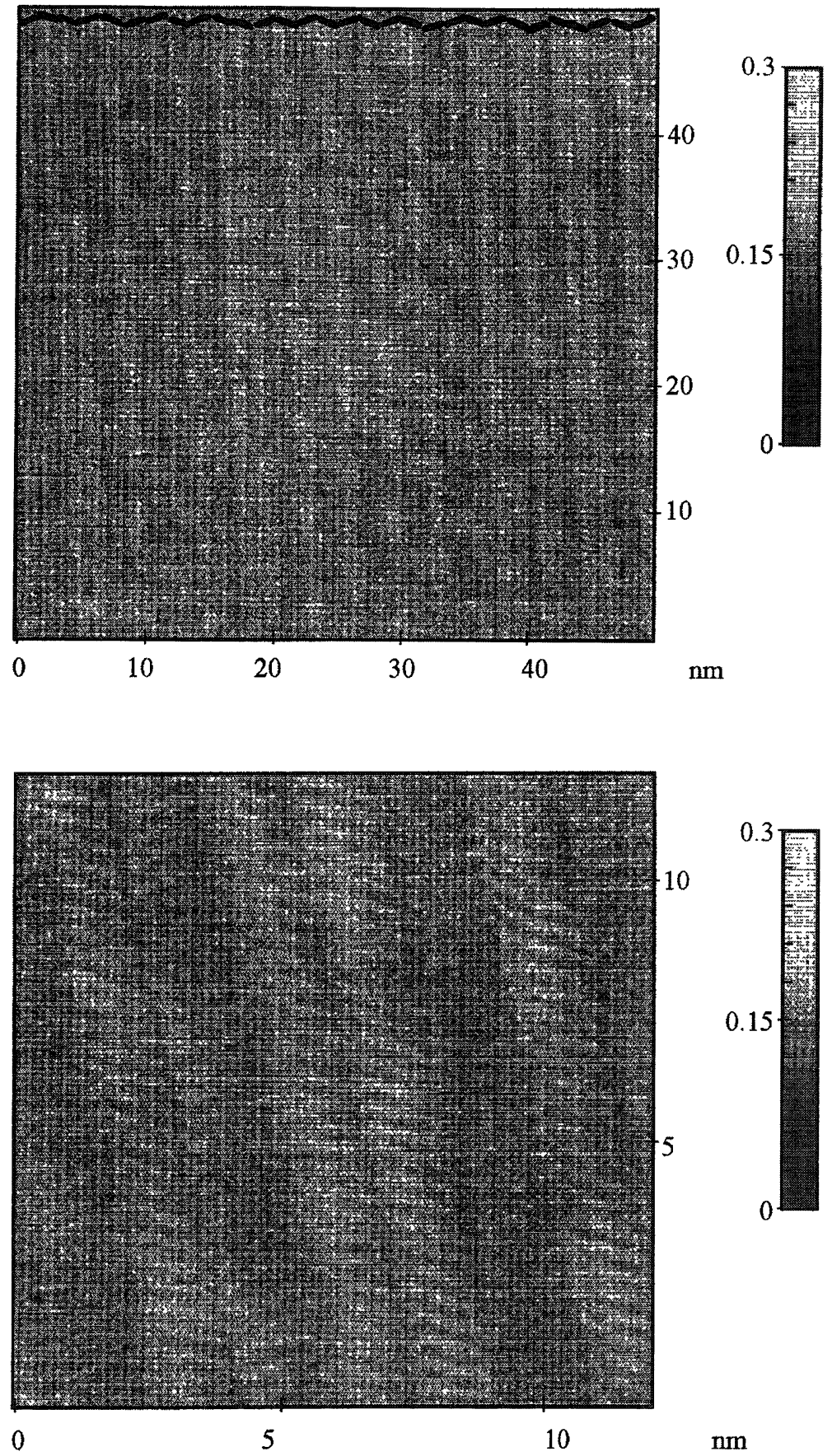

Fig. 1. - STM views of 1-dodecanol on graphite, $50 \times 50 \mathrm{~nm}^{2}$ (a) $0.12 \mathrm{nA}, 750 \mathrm{mV}$ ) and $12 \times 12 \mathrm{~nm}^{2}$ (b) $0.10 \mathrm{nA}, 750 \mathrm{mV}$ ). Molecular orientations are marked in a). 
organic adsorbates. In the case of significants defects (not singular ones), it may be an invaluable tool for the determination of the structure at liquid-solid interfaces. The direct molecular visualization opens a way to reach phenomena that are not easy to attain in far field techniques. A special interest is in the determination of randomly distributed but reproducible events. We will describe here a STM investigation of a 1-dodecanol layer adsorbed on graphite. The study was directed to the analysis of the defects of the self ordered structure.

\section{1-dodecanol.}

STM images of dodecanol $\left\{\mathrm{CH}_{3}-\left(\mathrm{CH}_{2}\right)_{11}-\mathrm{OH}\right.$, symbolized on schemes as $\left.-\mathrm{O}\right\}$, adsorbed on graphite (HOPG) are easily obtained. This work was done at room temperature $\left(\approx 20^{\circ} \mathrm{C}\right)$, with the adsorbate (mp: $24^{\circ} \mathrm{C}$ ) layer maintained liquid by a solvent (isopropanol or water traces). Observations were done with the tungsten tip within a thick layer of 1-dodecanol (a few microns). Tunnel conditions are not critical for contrast of images, for values of tunnel current from 0.1 to $0.5 \mathrm{nA}$, and bias voltage between 600 and $1000 \mathrm{mV}$.

The STM yields images of a well organized surface on large areas (fields of more than $250 \times$ $250 \mathrm{~nm}^{2}$ without domain boundary). Up to a $50 \times 50 \mathrm{~nm}^{2}$ field, molecules are individually seen (Fig. 1). The molecules are lying parallel to the graphite surface in a two-dimensional array, with an orientation angle of $\approx 60^{\circ}$ with lamellae edge. A moiré is also seen; it may be due to incommensurateness between adsorbate and substrate lattices, but its contrast is also dependent on tunnel conditions and sample area (presence of defects). These observations are similar to other recent works on the structure of alkanols on graphite, using STM [3-5] or X-ray diffraction [6].

An interesting STM study of dodecanol has been performed at various temperatures [5]. According to the sample temperature, two molecular arrays are described (shown in Fig. 2). At low temperature $\left(30 \sim 35^{\circ} \mathrm{C}\right)$, molecules are packed in a herringbone structure (a); after a phase transition, at $40{ }^{\circ} \mathrm{C}$ all molecules turned in a colinear arrangement between lamellae (b). The structure (b) is similar to the dodecanol bulk crystal.

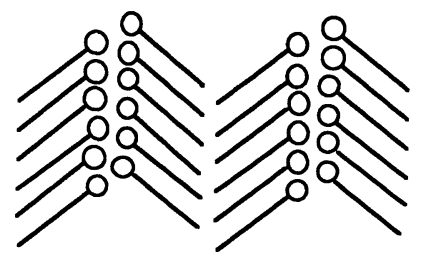

a)

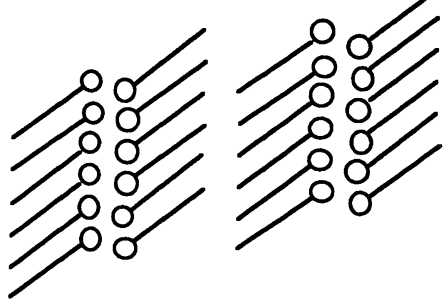

b)

Fig. 2. - Arrays of 1-dodecanol molecules adsorbed on graphite (from Ref. [5]) a) $t=30 \sim 35{ }^{\circ} \mathrm{C}$ b) $t=40^{\circ} \mathrm{C}$.

Significant information on the molecular arrangements of dodecanol is given by a careful analysis of the visualized fields with molecular resolution, on several samples. First, the actual structure is not exclusively in a herringbone array, as some vicinal lamellae made of colinear molecules are found easily (Fig. 1; such a defect is also found in Fig. 1a of Ref. [5]). Interestingly, the colinear lamellae are always going by two, separated by at least one (or uneven) angular interface from another such pair of lamellae. 
1)

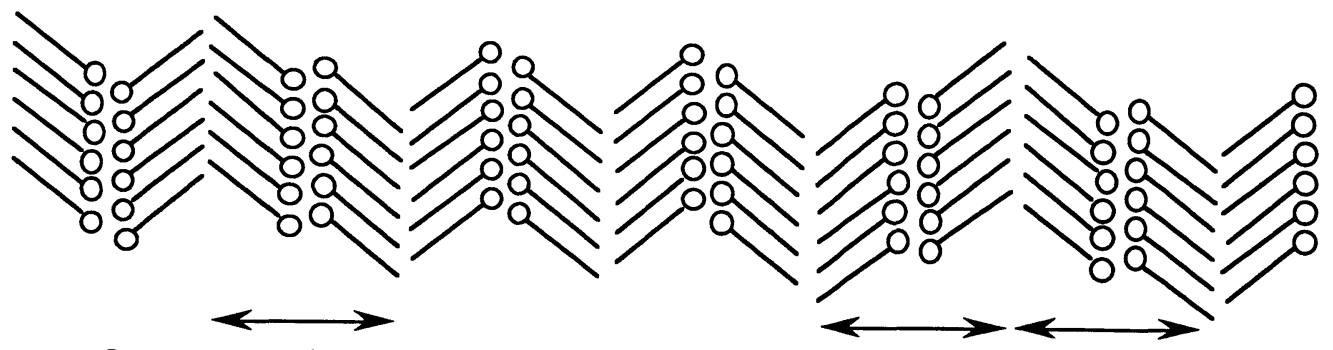

2)
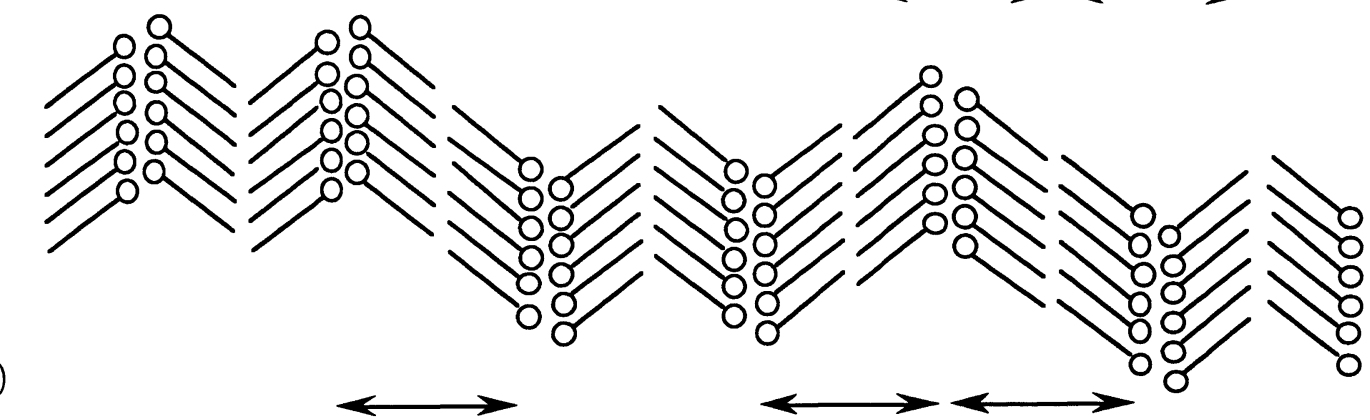

Fig. 3. - Molecular structures of 1-dodecanol adsorbed on graphite, from STM observations 1) Defects at the -OH ...HO- termini of dodecanol molecules (may be seen as (b) bands inserted in a (a) array) 2) Defects at the -Me Me- termini of dodecanol molecules (may be seen as crystalline defects in a pure (a) array).

The obvious consequence of this fact is that all irregular (colinear) junctions are in the same situation: methyl-methyl or hydroxyl-hydroxyl termini of dodecanol molecules, as the structure 1 or 2 shown in figure 3. The colinear pairs of lamellae are randomly distributed on the observed areas, with a likehood of $10 \sim 12 \%$ of all the interfaces (e.g. $20 \sim 25 \%$ of the Me-Me or OH-HO involved interfaces) [7].

As hydrogen bonds between hydroxyl groups yield more stabilization energy than interactions at the methyl end of molecules, case 1 (Fig. 3) seems inconsistent with the observation of no more than two vicinal colinear lamellae. In contrast, an arrangement with all the -OH $\cdots \mathrm{HO}$ - involved interfaces in the same conformation is more satisfactory. A screening of the tunnelling parameters (tunnel current: 0.01 to $0.5 \mathrm{nA}$ and voltage: -400 to $1000 \mathrm{mV}$ ) was not sufficient to distinguish unambiguously between both molecules termini.

Since a direct characterization was not completed by STM of a pure dodecanol layer, both ends of dodecanol molecules must be revealed indirectly. The observation of a layer of dodecanol coadsorbed on graphite with a suitable product was then considered. This coadsorbate must have certain characteristics in order to fit with dodecanol:

- chemical similarity to help coadsorption (no segregation)

- linear molecule with both ends identical

- coadsorbates easy to distinguish (by some geometrical factors as length)

Experiments were first done with 1,5-pentanediol $\left(\mathrm{mp}-18{ }^{\circ} \mathrm{C}\right)$, with no success. Alignments were seen with difficulty, whereas a molecular resolution must be obtained to observe the arrangement. The lack of sharpness in the STM images is likely due to the motion of molecules during observation of such short molecules at a temperature of $\approx 40^{\circ} \mathrm{C}$ above the melting point of the pure 1,5-pentanediol. 


\section{1-dodecanol dissolved in $\mathbf{1 , 8 - o c t a n e d i o l . ~}$}

The 1,8-octanediol (molecular length $\approx 1,3 \mathrm{~nm}$ ) was tested in several ratio with dodecanol (molecular length $\approx 1,7 \mathrm{~nm}$ ). Interesting images were obtained with a mixture of $\approx 20 \%$ dodecanol in $\approx 80 \%$ octanediol, pairs of dodecanol lamellae are seen dispersed into the octanediol matrix. We looked for the case where the expected defects are also present in the coadsorbate layer. Two situations have to be considered, relating to the arrangements of pure dodecanol; these areschematized in figure $4(\mathrm{O}-\mathrm{O}$ stands for 1,8-octanediol). If the defects are at the $-\mathrm{OH} \cdots \mathrm{HO}$ - junction, the presence of such colinear arrangements is highly likely between lamellae ofoctanediol molecules. All the observed interfaces between octanediol lamellae were found in angular (or normal) pattern. Figure 5 shows unfiltered STM images of $100 \times 100 \mathrm{~nm}^{2}$ (a) and $25 \times 25 \mathrm{~nm}^{2}$ (b) fields of dodecanol coadsorbed with octanediol. With a scale of $100 \times 100 \mathrm{~nm}^{2}$, only lamellae are distinguished, displaying the good dispersion of dodecanol (see arrows in Fig. 5a). A $25 \times 25 \mathrm{~nm}^{2}$ area (Fig. 5a) is shown with molecular resolution, all interfaces are regular. In such a view, since the molecular ends of dodecanol are known, the junction inside a pair of dodecanol lamellae has to be $\mathrm{Me} \mathrm{Me} \mathrm{(see} \mathrm{arrows} \mathrm{in} \mathrm{Fig.} \mathrm{5b).} \mathrm{Notice} \mathrm{the} \mathrm{lateral} \mathrm{sliding} \mathrm{of} \mathrm{lamellae} \mathrm{in} \mathrm{the} \mathrm{central} \mathrm{part} \mathrm{of} \mathrm{view}$ (Fig. 5, arrows at both ends). An area of $15 \times 15 \mathrm{~nm}^{2}$ with the desired defects is presented in figure 6. On this crude high resolution STM image, the molecular orientations are schematized for interesting lamellae. It is easy to see the presence of colinear junction inside the dodecanol pair, revealing that defects are located at the Me Me molecular interface (as in Fig. 4.2). With a good probability, the defects of the pure dodecanol layer can be considered in the same situation, as schematized in figure 3.2.
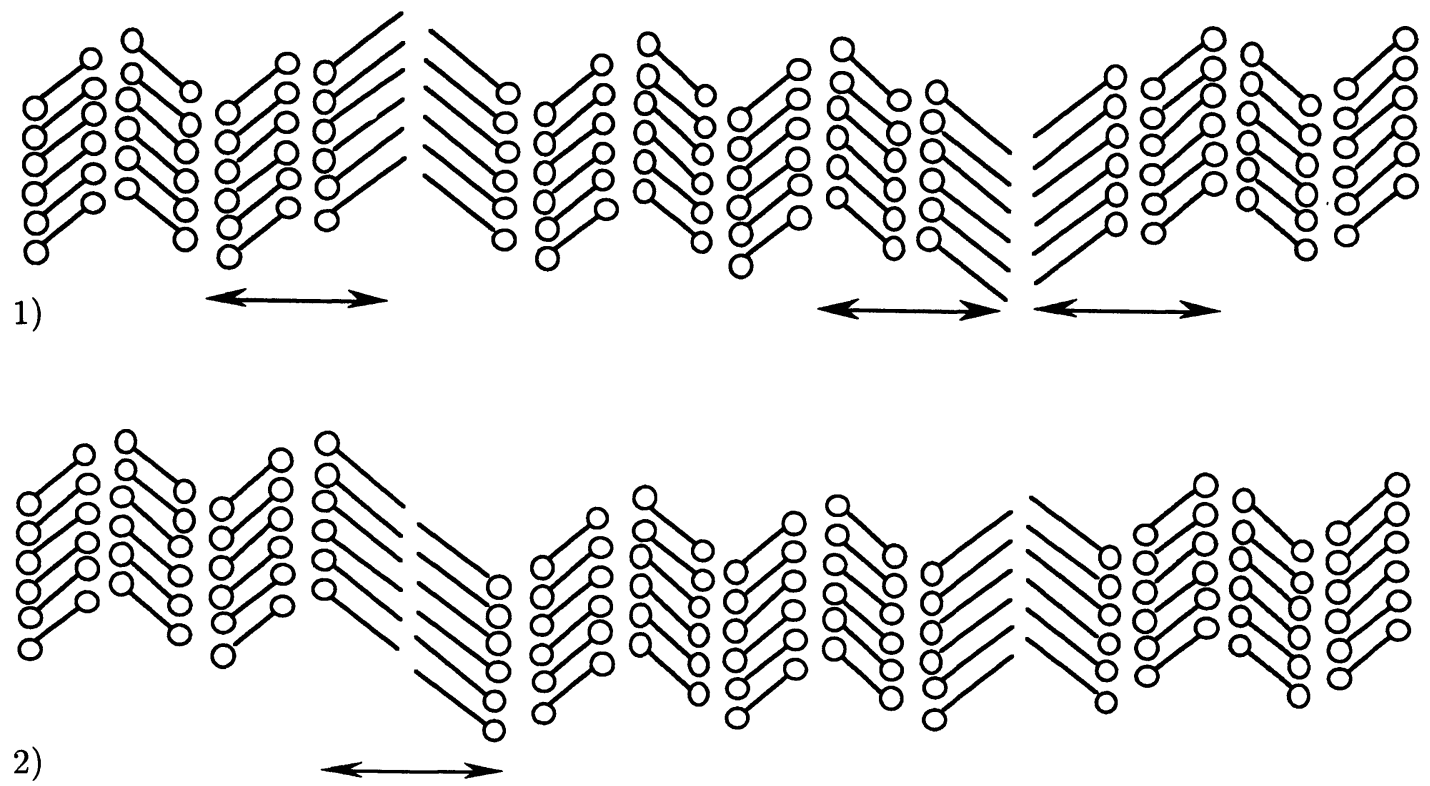

Fig. 4. - Molecular structures of 1-dodecanol-1,8-octanediol coadsorbed on graphite 1) Defects at the -OH . . HO- termini of dodecanol molecules, 2) Defect at the -Me Me-termini of dodecanol molecules. 

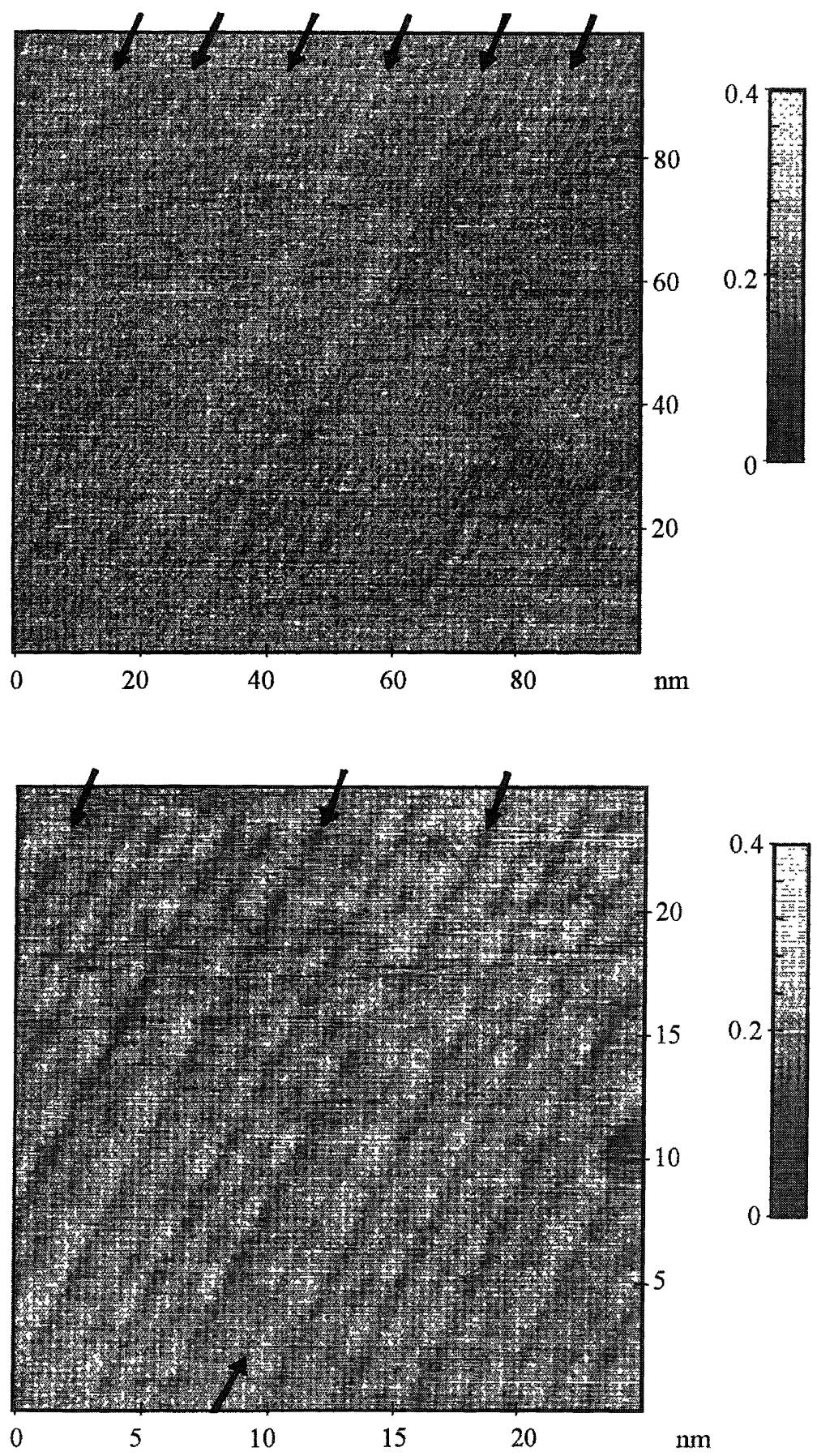

Fig. 5. - STM images of $100 \times 100 \mathrm{~nm}^{2}$ (a) $0.12 \mathrm{nA} 1450 \mathrm{mV}$ ) and $25 \times 25 \mathrm{~nm}^{2}$ (b) $0.08 \mathrm{nA} 1315 \mathrm{mV}$ ) fields of 1-dodecanol coadsorbed with 1,8-octanediol. Arrows show dodecanol lamellae, in $b$ ) notice the lateral sliding of lamellae marked with two arrows. 


\section{Conclusion.}

The STM analysis of a layer of pure dodecanol adsorbed on graphite revealed the presence of one class of structure defects. The study of coadsorbed dodecanol-octanediol was found to be complementary to the first one and allowed the nature of the structural defects to be elucidated.

The knowledge of structures at liquid-solid interface is of great interest, as in moistening, coating, lubrication and adhesion sciences.

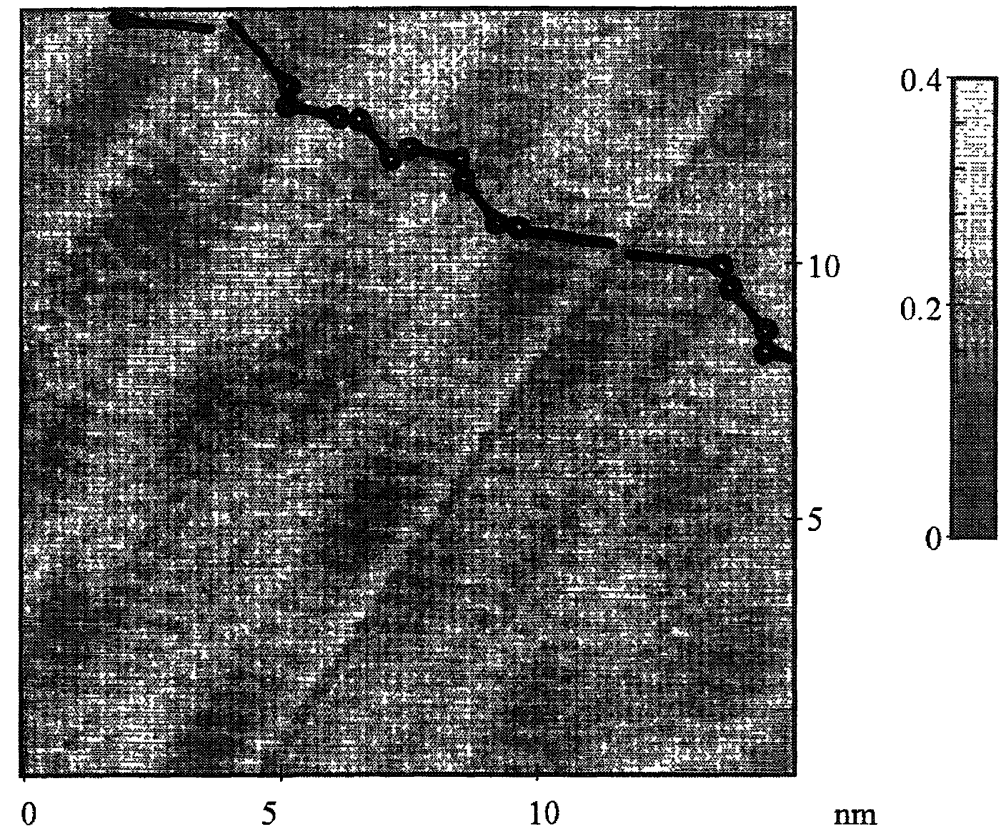

Fig. 6. - STM view of $15 \times 15 \mathrm{~nm}^{2}$ area with colinear lamellae of dodecanol, coadsorbed with octanediol $(0,10 \mathrm{nA}, 1200 \mathrm{mV})$. Molecular orientations are shown.

\section{Experimental.}

Chemicals (1-dodecanol, 1,5-pentanediol and 1,8-octanediol) were purchased from Fluka (Buchs, Switzerland), solvents (iPrOH and ethanol) were obtained from Carlo Erba (Milano, Italia); all solvents and chemicals were used without further purification. Graphite (HOPG) was purchased from Le Carbone Lorraine (Gennevilliers, France).

STM experiments were done on a Nanoscope II (from Digital Instruments, Santa Barbara, California) equipped with electrochemically etched tungsten lab-made tips (wire diameter $0.2 \mathrm{~mm}$, etched in $1 \mathrm{~N} \mathrm{NaOH}$ in ethanol-water solution). An "A" head (maxi scan area $660 \times 660 \mathrm{~nm}^{2}$ ) was used, equipped with a low noise preamplifier.

Samples were prepared by spreading of a drop of the alkanol onto a small piece of HOPG. The layer of adsorbate was maintained liquid, generally by adding isopropanol or by melting near 
a heat source (office lamp for dodecanol), to avoid crashing the tip by contact with crystalline deposit. Images were obtained in the constant current mode (or height mode), under usual tunnelling conditions. For pure dodecanol, tunnel current from 0.01 to $0.5 \mathrm{nA}$ and biases of -400 to $1000 \mathrm{mV}$ were tested, whereas useful values are $0.1 \sim 0.5 \mathrm{nA}$ and $600 \sim 1000 \mathrm{mV}$. The observation of dodecanol-pentanediol was performed using $0.15 \sim 0.2 \mathrm{nA}$ and $740 \sim 1400 \mathrm{mV}$, and the imaging of dodecanol-octanediol blend was done in $0.04 \sim 0.12 \mathrm{nA}$ and $1090 \sim 1450 \mathrm{mV}$ tunnel conditions.

\section{Acknowledgements.}

We are obliged to CNRS, MRES and Conseil Général de l'Essonne for their financial support. Prof. H.B. Kagan is acknowledged for many stimulating discussions.

\section{References}

[1] Frommer J., Angew. Chem. Int. Ed. Engl. 31 (1992) 1298-1328.

[2] Fuchs H., J. Molec. Struct. 292 (1993) 29-48.

[3] Buchholz S. and Rabe J.P., Angew. Chem. Int. Ed. Eng. 31 (1992) 189.

[4] Yeo Y.H., Yakoboski K., McGonigal G.C. and Thomson D.J., J. Vac. Sci. Technol. 10 (1992) 600; Ultramicroscopy 42-44 (1992) 963.

[5] Yeo Y.H., McGonigal G.C. and Thomson D.J., Langmuir 9 (1993) 649-651.

[6] Morishige K., Takami Y. and Yokota Y., Phys. Rev. B 48 (1993) 8277.

[7] For a preliminary report, on pure dodecanol imaging, see: J-C. Poulin, Analusis 22 (1994) M41-43. 